\title{
Influência da Chuva na Eficiência de Diquat no Controle de Salvinia auriculata, Pistia stratiotes E Eichhornia crassipes ${ }^{1}$
}

\author{
Influence of Rainfall on Diquat Efficiency in Controlling Salvinia auriculata, Pistia stratiotes \\ and Eichhornia crassipes
}

\author{
SOUZA, G.S.F. ${ }^{2}$, CAMPOS, C.F. ${ }^{3}$, PEREIRA, M.R.R. ${ }^{4}$ e MARTINS, D. ${ }^{5}$
}

\begin{abstract}
RESUMO - O objetivo deste estudo foi avaliar a influência da ocorrência de chuva em diferentes períodos de tempo após a aplicação do herbicida diquat, em sua eficiência de controle sobre plantas de Salvinia auriculata, Pistia stratiotes e Eichhornia crassipes. Foram estudadas chuvas de $10 \mathrm{~mm}$, aplicadas durante $5 \mathrm{~min}$, em diferentes periodos de tempo $(0 \mathrm{~h}, 0,25 \mathrm{~h}, 0,5 \mathrm{~h}, 1 \mathrm{~h}, 2 \mathrm{~h}$, 4h, 6h, 12h e não simulação de chuva) após a aplicação de diquat, na formulação Reward, a 600 g i.a. ha ${ }^{-1}$ (2,5 L p.c. ha $\left.{ }^{-1}\right)$. O delineamento experimental utilizado foi o inteiramente casualizado, com quatro repetições. As plantas foram avaliadas visualmente aos 3, 5, 7 e 12 dias após a aplicação (DAA), sendo os resultados submetidos à análise de variância pelo teste F, e as médias dos tratamentos, comparadas pelo teste de Tukey $(\mathrm{p}<0,05)$. O herbicida diquat proporcionou excelente controle de todas as espécies estudadas, independentemente do período de tempo para ocorrência de chuva depois de sua aplicação, até mesmo com ocorrência de chuva imediatamente após sua aplicação.
\end{abstract}

Palavras-chave: planta daninha aquática, aguapé, alface-d'água, herbicida.

\begin{abstract}
The aim of this study was to evaluate the influence of rainfall events at different time periods after application of diquat on the efficiency of this herbicide in controlling Salvinia auriculata, Pistia stratiotes and Eichhornia crassipes. Simulation of $10 \mathrm{~mm}$ rainfall applied during 5 min was studied at different time periods $(0 h, 0.25 \mathrm{~h}, 0.5 \mathrm{~h}, 1 \mathrm{~h}, 2 \mathrm{~h}, 4 \mathrm{~h}, 6 \mathrm{~h}, 12 \mathrm{~h}$ and no rain

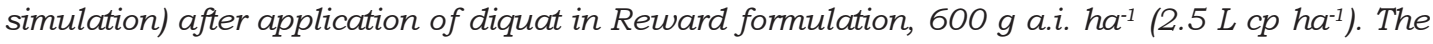
experiment was arranged in a completely randomized design with four replications. Plants were evaluated at 3, 5, 7, and 12 days after application (DAA), and the results submitted to analysis of variance by the $F$ test and the treatment means compared by the Tukey test ( $p<0.05)$. Diquat provided excellent control of all the species studied, regardless of the time period for rain occurrence after application, even when immediately after application.
\end{abstract}

Keywords: aquatic weed, water hyacinth, water lettuce, herbicide.

\section{INTRODUÇÃO}

Lagos e reservatórios são fundamentais para o fornecimento de água a diversos sistemas - entre estes, o agrícola. No entanto, a intervenção antrópica estabelecida de maneira não planejada sobre os diversos ecossistemas aquáticos - como a construção de reservatórios para fins hidrelétricos, fonte de água potável para a população urbana ou para irrigação de lavouras, a manipulação dos níveis higrométricos desses reservatórios, o aquecimento global, a eutrofização e o desmatamento das matas ciliares - promove uma

Recebido para publicação em 12.5.2010 e aprovado em 25.2.2011.

2 Doutorando em Agronomia/Agricultura, Faculdade de Ciências Agronômicas, Universidade Estadual Paulista Júlio de Mesquita Filho - FCA/UNESP, Caixa Postal 237, 18603-970 Botucatu-SP, <guisasso@hotmail.com>; ${ }^{3}$ Mestrando em Agronomia/Agricultura, FCA-UNESP, Botucatu-SP; ${ }^{4}$ Pós-doutoranda em Agronomia, FCA-UNESP; ${ }^{5}$ Professor Adjunto do Dep. de Produção Vegetal, FCA/UNESP, Botucatu-SP. 
série de alterações nas características químicas, físicas e bióticas dos corpos hídricos, levando a mudanças expressivas na comunidade biótica que os coloniza, incluindo a expansão de populações de macrófitas aquáticas (Campos et al., 2010; Cancian et al., 2009; Marchi, 2006).

As macrófitas aquáticas possuem papel fundamental no estoque de energia e carbono nas bases das pirâmides alimentares, promovem habitats adequados para muitos organismos e servem de suporte à oviposição e refúgio de muitos peixes e outros animais (Neves et al., 2002). Contudo, algumas macrófitas, por apresentarem elevada capacidade de multiplicação vegetativa, dominam o ambiente em que estão inseridas e causam uma série de problemas, sendo tituladas como plantas daninhas aquáticas.

Entre as plantas daninhas aquáticas, as flutuantes causam os mais difundidos problemas em nivel mundial. Elas normalmente apresentam independência das estruturas sexuais de reprodução, grande área de tecido fotossintético em proporção ao comprimento da planta, capacidade rápida de ocupar locais disponiveis onde incida luz, reduzindo sua penetração e a capacidade fotossintética dos vegetais imersos, e elevação dos índices de evaporação da área infestada; ademais, dificultam a navegação, emperram equipamentos de irrigação, afetam o desempenho de usinas hidroelétricas e, até mesmo, abrigam animais indesejados e vetores de doenças, além da independência das condições do substrato, devido ao fluxo d'água e à localização das plantas (Kissmann, 1997; IBAMA, 1998).

Entre as principais plantas aquáticas flutuantes causadoras de danos e problemas aos ecossistemas aquáticos encontram-se a salvínia (Salvinia auriculata), a alface-d'água (Pistia stratiotes) e o aguapé (Eichhornia crassipes).

No Brasil, no momento, não há nenhum herbicida registrado para controle dessas três espécies (Motta et al., 2008). Em outros países, como Estados Unidos, Canadá e Nova Zelândia, as plantas aquáticas vêm sendo estudadas há muitos anos, e diferentes métodos de controle - mecânicos, biológicos e químicos - são adotados. Dentro do controle químico, uma grande quantidade de herbicidas já foi testada, sendo selecionados aqueles em que o controle não acarreta prejuízos ao habitat natural dessas plantas ou mesmo a peixes e outros organismos aquáticos (Marchi, 2006), como, por exemplo, o diquat, que é um herbicida muito usado no controle de plantas daninhas aquáticas nos Estados Unidos, sendo 60\% do total aplicado no estado da Flórida, que utiliza cerca de 50 mil litros por ano do produto formulado.

O diquat é um herbicida de contato, não seletivo, inibidor do fotossistema I e pertencente ao grupo químico dos bipiridílios; demonstra elevado potencial para uso em ambientes aquáticos no controle de plantas daninhas emersas e imersas, por ser pouco atraído aos materiais lipídicos, possuir baixas taxas de bioconcentração (Lavorenti, 1996; Rodrigues \& Almeida, 2005), dissipar-se rapidamente na água e por sua baixa toxicidade a organismos aquáticos (Ritter et al., 2000; Henares et al., 2007, 2008a, b).

No entanto, a eficiência de controle dos herbicidas pode ser influenciada pela ocorrência de chuvas após sua aplicação; uma chuva de baixa intensidade e curta duração pode proporcionar reumedecimento da gota e/ou redistribuição do herbicida na planta, aumentando a eficiência do produto aplicado (Jakelaitis et al., 2001). Contudo, de modo geral, chuvas logo após aplicação de herbicidas podem reduzir sua eficiência no controle das plantas, e fatores como intervalo de tempo e intensidade e duração da chuva após aplicação dos herbicidas, bem como condições climáticas antes das aplicações, interferem na eficiência dos herbicidas aplicados em pós-emergência (Anderson \& Arnold, 1984).

Em razão das poucas informações na literatura referentes ao período mínimo de tempo sem a ocorrência de chuva após a aplicação do herbicida diquat no controle de espécies daninhas aquáticas, torna-se fundamental a realização de estudos de controle químico com posterior simulação de chuva que possam auxiliar nas tomadas de decisão de manejo dessas espécies. O objetivo deste estudo foi avaliar a influência de diferentes períodos de chuva ocorridos após a aplicação do herbicida diquat em sua eficiência de controle sobre plantas de $S$. auriculata, $P$. stratiotes e E. crassipes. 


\section{MATERIAL E MÉTODOS}

O experimento foi conduzido em casa de vegetação no Núcleo de Pesquisas Avançadas em Matologia (NUPAM), pertencente ao Departamento de Produção Vegetal, setor de Agricultura, da Faculdade de Ciências Agronômicas/UNESP, campus de Botucatu/SP.

As plantas de $S$. auriculata, $P$. strstiotes e $E$. crassipes provenientes de uma represa localizada no municipio de Botucatu-SP foram conduzidas em vasos plásticos de $15 \times 15 x$ $15 \mathrm{~cm}$, com 2,5 litros de água, mantidos em casa de vegetação; em cada vaso, foi mantida apenas uma planta.

O experimento foi conduzido em delineamento totalmente casualizado, com quatro repetições; os tratamentos estudados constaram da simulação de uma chuva de $10 \mathrm{~mm}$ (aplicada durante $5 \mathrm{~min}$ ) em diferentes periodos de tempo (0h, 0,25h, 0,5h, 1h, 2h, 4h, 6h, 12h e não simulação de chuva) após a aplicação de diquat, na formulação Reward, a 600 g i.a. ha ${ }^{-1}$ (2,5 L p.c. ha $\left.{ }^{-1}\right)$.

A simulação da chuva foi feita sob um sistema estacionário de aplicação de água, regulado para a aplicação da chuva pretendida. Ressaltase que o período de $0 \mathrm{~h}$ para a simulação da chuva ocorreu em não mais que 30 segundos, sendo este o tempo necessário entre o término da pulverização do diquat e a partida do sistema estacionário de aplicação da chuva.

A aplicação do herbicida foi realizada por meio de um pulverizador costal, com pressão constante de $\mathrm{CO}_{2}$, consumo de calda de $200 \mathrm{~L} \mathrm{ha}^{-1}$ e barra de aplicação equipada com duas pontas tipo jato plano Teejet XR 11002VS, distanciadas de $0,50 \mathrm{~m}$ entre si.

As plantas foram avaliadas visualmente aos 3, 5, 7 e 12 dias após a aplicação (DAA), por meio de uma escala percentual de notas, em que zero representava nenhum controle e $100 \%$ o controle total das plantas, considerando-se eficazes os tratamentos que proporcionaram controle $\geq 80 \%$ (Sociedade Brasileira da Ciência das Plantas Daninhas, 1995). Os parâmetros utilizados no estabelecimento das notas visuais de controle foram: acúmulo de biomassa, inibição do crescimento, quantidade e uniformidade das injúrias e capacidade de rebrota das plantas.
Os resultados foram submetidos à análise de variância pelo teste $\mathrm{F}$, sendo as médias dos tratamentos comparadas pelo teste de Tukey $(\mathrm{p}<0,05)$.

\section{RESULTADOS E DISCUSSÃO}

Observa-se, na Tabela 1, que aos 3 DAA nenhum dos tratamentos apresentou controle considerado satisfatório sobre as plantas de $S$. auriculata, e os diferentes intervalos de tempo de chuva não provocaram diferenças na eficiência de controle do herbicida.

$\mathrm{Na}$ avaliação realizada aos 5 DAA, nota-se que houve incremento no controle das plantas em todos os tratamentos estudados; chuvas a partir de uma hora da aplicação do herbicida não interferiram no seu desempenho. Entretanto, biologicamente, nesta avaliação, apenas o tratamento com ocorrência de chuva quatro horas após a aplicação de diquat exerceu controle considerado satisfatório das plantas de salvínia, com $82,5 \%$.

Aos 7 DAA, foi possivel registrar que a chuva ocorrida após meia hora da aplicação do herbicida também passou a exercer controle semelhante ao das chuvas ocorridas posteriormente. No entanto, apenas os tratamentos com chuvas após 4, 6 e 12 horas da

Tabela 1 - Porcentagem de controle de plantas de Salvinia auriculata com o herbicida diquat (600 g i.a. ha-1) após aplicação dos tratamentos químicos, sob diferentes intervalos sem chuva. Botucatu-SP, 2009

\begin{tabular}{|c|c|c|c|c|}
\hline \multirow{2}{*}{$\begin{array}{c}\text { Período sem } \\
\text { chuva (horas) }\end{array}$} & \multicolumn{4}{|c|}{ Dias após a aplicação (DAA) } \\
\cline { 2 - 5 } & 3 & 5 & 7 & 12 \\
\hline 0 & $10,50 \mathrm{~A}$ & $36,25 \mathrm{C}$ & $47,50 \mathrm{~B}$ & $99,75 \mathrm{~A}$ \\
\hline 0,25 & $10,50 \mathrm{~A}$ & $35,00 \mathrm{C}$ & $45,75 \mathrm{~B}$ & $100,00 \mathrm{~A}$ \\
\hline 0,5 & $10,50 \mathrm{~A}$ & $51,25 \mathrm{BC}$ & $66,75 \mathrm{AB}$ & $100,00 \mathrm{~A}$ \\
\hline 1 & $11,00 \mathrm{~A}$ & $56,25 \mathrm{ABC}$ & $73,25 \mathrm{AB}$ & $100,00 \mathrm{~A}$ \\
\hline 2 & $10,00 \mathrm{~A}$ & $55,00 \mathrm{ABC}$ & $71,75 \mathrm{AB}$ & $100,00 \mathrm{~A}$ \\
\hline 4 & $10,00 \mathrm{~A}$ & $82,50 \mathrm{~A}$ & $100,00 \mathrm{~A}$ & $100,00 \mathrm{~A}$ \\
\hline 6 & $10,00 \mathrm{~A}$ & $67,50 \mathrm{AB}$ & $88,00 \mathrm{~A}$ & $100,00 \mathrm{~A}$ \\
\hline 12 & $10,00 \mathrm{~A}$ & $73,75 \mathrm{AB}$ & $92,00 \mathrm{~A}$ & $100,00 \mathrm{~A}$ \\
\hline Sem chuva & $10,00 \mathrm{~A}$ & $57,50 \mathrm{ABC}$ & $72,25 \mathrm{AB}$ & $100,00 \mathrm{~A}$ \\
\hline Frtatamento $_{-1}$ & $0,090^{\text {ns }}$ & $6,406 *$ & $6,239^{* *}$ & $0,500^{\text {ns }}$ \\
\hline CV $(\%)$ & 23,6 & 21,8 & 20,3 & 0,4 \\
\hline d.m.s. & 5,76 & 29,68 & 35,33 & 0,89 \\
\hline
\end{tabular}

Médias seguidas da mesma letra maiúscula na coluna não diferem estatisticamente entre si pelo teste de Tukey $(p<0,05)$. ** Significativo a $1 \%$ de probabilidade. ${ }^{\text {ns }}$ não significativo. 
aplicação apresentaram controles que variaram de bom a excelente da planta daninha.

Já na última avaliação, aos 12 DAA, verificou-se que todos os tratamentos estudados apresentaram controle excelente da planta daninha. Martins et al. (2002), estudando o efeito do diquat aplicado em plantas de S. molesta sem ocorrência de precipitações após sua aplicação, observaram controle excelente a partir de 11 DAA. Assim, registrouse que a eficiência de controle do herbicida diquat aplicado sobre plantas de $S$. auriculata não foi influenciada negativamente por nenhum dos intervalos de tempo de chuva ocorridos após a sua aplicação; o controle foi de 99,25\% quando da ocorrência de chuva imediatamente após sua aplicação e total nos demais tratamentos estudados.

Observa-se na Tabela 2 que, aos 3 DAA, todos os tratamentos proporcionaram efeitos tóxicos às plantas de $P$. stratiotes; a eficiência de controle do diquat decresceu com a proximidade das chuvas após a aplicação do herbicida, com diferenças de até $67,5 \%$ no controle da planta daninha, quando comparado o primeiro momento de simulação de chuva ao tratamento sem chuva.

Já aos 5 DAA, os tratamentos passaram a controlar eficientemente a planta daninha, porém nenhum atingiu ainda o controle total dela; o tratamento com simulação de chuva imediatamente após a aplicação do diquat apresentou controle considerado bom. Martins et al. (2002), utilizando também diquat no controle da mesma planta daninha aquática, mas sem a ocorrência de chuva, observaram controle máximo de alface-d'água nesse mesmo periodo de avaliação - fato que demonstra certo atraso na ação desse herbicida quando da ocorrência de chuvas.

Aos 7 DAA, o controle das plantas de $P$. stratiotes continuou aumentando e alcançou o nível máximo em quase todos os tratamentos, sendo menor no tratamento com chuva simulada imediatamente após a aplicação do herbicida, porém com controle também considerado excelente.

Na última avaliação, aos 12 DAA, nota-se que todos os tratamentos com ocorrência de chuva após 0,25 hora (15 minutos) da aplicação do herbicida diquat controlaram totalmente as plantas, e, quando da ocorrência de chuva imediatamente após a aplicação do herbicida o controle foi também eficiente, demonstrando assim que o herbicida diquat não foi influenciado pela ocorrência de chuvas nos diversos períodos de tempo estudados após sua aplicação. Esses resultados de controle foram semelhantes aos obtidos por Langeland et al. (2002), que relataram a elevada eficiência de diquat no controle de plantas de $P$. stratiotes aos 14 DAA com uso de doses que variaram de 2,3 a $7,0 \mathrm{~L}$ p.c. ha ${ }^{-1}$, sem posterior ocorrência de chuva após sua aplicação.

Registra-se na Tabela 3 que todos os tratamentos estudados proporcionaram efeitos tóxicos às plantas de E. crassipes; já aos 3 DAA, como observado para S. auriculata e $P$. stratiotes, esses efeitos visuais foram intensos com o aumento do período de exposição das plantas ao herbicida diquat sem a ocorrência de precipitações, passando de $17,0 \%$ para $77,5 \%$ o controle das plantas quando da ausência de chuva em relação à ocorrência de chuva no momento da aplicação, o que corrobora Neves et al. (2002), que, ao estudarem os efeitos do diquat (na dose de 750 g i.a. ha ${ }^{-1}$ ) sobre a mesma espécie, observaram controles bem semelhantes sem a ocorrência de chuva.

Tabela 2 - Porcentagem de controle de plantas de Pistia stratiotes com o herbicida diquat (600 g i.a. ha ${ }^{-1}$ ) após aplicação dos tratamentos químicos, sob diferentes intervalos sem chuva. Botucatu-SP, 2009

\begin{tabular}{|c|c|c|c|c|}
\hline \multirow{2}{*}{$\begin{array}{c}\text { Período sem } \\
\text { chuva (horas) }\end{array}$} & \multicolumn{4}{|c|}{ Dias após a aplicação (DAA) } \\
\cline { 2 - 5 } & 3 & \multicolumn{1}{c|}{5} & \multicolumn{1}{c|}{7} & 12 \\
\hline 0 & $27,50 \mathrm{E}$ & $82,50 \mathrm{~B}$ & $97,50 \mathrm{~B}$ & $98,50 \mathrm{~B}$ \\
\hline 0,25 & $45,00 \mathrm{D}$ & $92,00 \mathrm{~A}$ & $100,00 \mathrm{~A}$ & $100,00 \mathrm{~A}$ \\
\hline 0,5 & $54,50 \mathrm{CD}$ & $95,00 \mathrm{~A}$ & $100,00 \mathrm{~A}$ & $100,00 \mathrm{~A}$ \\
\hline 1 & $58,75 \mathrm{C}$ & $96,75 \mathrm{~A}$ & $99,75 \mathrm{~A}$ & $100,00 \mathrm{~A}$ \\
\hline 2 & $81,25 \mathrm{~B}$ & $97,25 \mathrm{~A}$ & $100,00 \mathrm{~A}$ & $100,00 \mathrm{~A}$ \\
\hline 4 & $81,35 \mathrm{~B}$ & $98,00 \mathrm{~A}$ & $100,00 \mathrm{~A}$ & $100,00 \mathrm{~A}$ \\
\hline 6 & $82,50 \mathrm{AB}$ & $97,50 \mathrm{~A}$ & $100,00 \mathrm{~A}$ & $100,00 \mathrm{~A}$ \\
\hline 12 & $88,25 \mathrm{AB}$ & $98,75 \mathrm{~A}$ & $99,50 \mathrm{~A}$ & $100,00 \mathrm{~A}$ \\
\hline Sem chuva & $95,00 \mathrm{~A}$ & $99,75 \mathrm{~A}$ & $100,00 \mathrm{~A}$ & $100,00 \mathrm{~A}$ \\
\hline F $_{\text {tratamento }}$ & $73,736^{* *}$ & $9,328^{* *}$ & $4,343^{* *}$ & $3,000^{*}$ \\
\hline CV $\%$ ) & 7,8 & 3,6 & 0,8 & 0,6 \\
\hline d.m.s. & 12,59 & 8,26 & 1,87 & 1,37 \\
\hline
\end{tabular}

Médias seguidas da mesma letra maiúscula na coluna não diferem estatisticamente entre si pelo teste de Tukey $(p<0,05)$. ** Significativo a $1 \%$ de probabilidade. * Significativo a $5 \%$ de probabilidade. 
Aos 5 DAA, os efeitos do herbicida diquat sobre as plantas elevaram-se em todos os tratamentos e a ação da chuva na eficiência do diquat continuou a ser observada, o que afetou negativamente seu desempenho no controle das plantas de aguapé, com a aproximação da chuva à aplicação do herbicida. Os tratamentos com ocorrência de chuvas após meia hora da aplicação do herbicida mostraram-se eficientes no controle da planta aquática, com controle acima de $81,0 \%$. Cardoso et al. (2003), trabalhando também com o herbicida diquat aplicado em diferentes acessos de aguapé, mas sem a ocorrência de chuvas após a aplicação, observaram porcentagens de controle desta espécie entre $95 \%$ e $99 \%$, fato que confirma a influência negativa da chuva na ação do herbicida, porém não em níveis abaixo do que é considerado satisfatório. No estudo realizado por Neves et al. (2002), observou-se que a aplicação de diquat na dose de 400 g i.a. ha ${ }^{-1}$, no controle de plantas de aguapé submetidas à simulação de chuva de 6,92 $\mathrm{mm}$ após meia hora da aplicação, apresentou controle considerado bom $(86,67 \%)$ somente após 20 DAA.

$\mathrm{Na}$ avaliação posterior, aos 7 DAA, foi possivel observar que o herbicida diquat passou a ser ainda mais eficiente no controle das

Tabela 3 - Porcentagem de controle de plantas de Eichhornia crassipes com o herbicida diquat (600 g i.a. ha-1) após aplicação dos tratamentos químicos, sob diferentes intervalos sem chuva. Botucatu-SP, 2009

\begin{tabular}{|c|c|c|c|c|}
\hline \multirow{2}{*}{$\begin{array}{c}\text { Período sem } \\
\text { chuva (horas) }\end{array}$} & \multicolumn{4}{|c|}{ Dias após a aplicação (DAA) } \\
\cline { 2 - 5 } & 3 & 5 & 7 & 12 \\
\hline 0 & $17,00 \mathrm{~F}$ & $41,25 \mathrm{C}$ & $51,15 \mathrm{~B}$ & $92,50 \mathrm{~A}$ \\
\hline 0,25 & $27,50 \mathrm{EF}$ & $75,75 \mathrm{~B}$ & $84,75 \mathrm{~A}$ & $92,50 \mathrm{~A}$ \\
\hline 0,5 & $32,00 \mathrm{E}$ & $81,75 \mathrm{AB}$ & $83,00 \mathrm{~A}$ & $90,00 \mathrm{~A}$ \\
\hline 1 & $50,00 \mathrm{CD}$ & $91,50 \mathrm{AB}$ & $94,00 \mathrm{~A}$ & $97,50 \mathrm{~A}$ \\
\hline 2 & $46,25 \mathrm{D}$ & $92,50 \mathrm{AB}$ & $93,75 \mathrm{~A}$ & $95,00 \mathrm{~A}$ \\
\hline 4 & $60,00 \mathrm{BC}$ & $95,50 \mathrm{~A}$ & $96,50 \mathrm{~A}$ & $100,00 \mathrm{~A}$ \\
\hline 6 & $61,25 \mathrm{BC}$ & $93,50 \mathrm{AB}$ & $96,75 \mathrm{~A}$ & $97,00 \mathrm{~A}$ \\
\hline 12 & $66,50 \mathrm{AB}$ & $96,00 \mathrm{~A}$ & $97,00 \mathrm{~A}$ & $98,50 \mathrm{~A}$ \\
\hline Sem chuva & $77,50 \mathrm{~A}$ & $98,50 \mathrm{~A}$ & $98,50 \mathrm{~A}$ & $100,00 \mathrm{~A}$ \\
\hline F $_{\text {tratamento }}$ & $63,683^{* *}$ & $19,315^{* *}$ & $11,343 * *$ & $0,425^{\text {ns }}$ \\
\hline CV $(\%)$ & 10,3 & 9,7 & 10,1 & 11,6 \\
\hline d.m.s. & 11,88 & 19,50 & 21,16 & 26,26 \\
\hline
\end{tabular}

Médias seguidas da mesma letra maiúscula na coluna não diferem estatisticamente entre si pelo de teste Tukey $(p<0,05)$. ** Significativo a $1 \%$ de probabilidade. ${ }^{\text {ns }}$ não significativo. plantas de aguapé em todos os tratamentos que receberam chuva após sua aplicação. Entretanto, diferentemente da avaliação anterior, o herbicida mostrou-se eficiente no controle da planta daninha até mesmo com a ocorrência de chuvas a partir de 15 minutos da sua aplicação.

Na última avaliação visual, aos 12 DAA, notou-se que, estatisticamente, todos os períodos de tempo de chuva estudados passaram a não influenciar negativamente a ação do herbicida diquat sobre as plantas de $E$. crassipes; contudo, em termos biológicos, o controle observado para os tratamentos que receberam chuvas até meia hora após a aplicação do herbicida diquat pode ser apenas considerado bom, diferentemente dos demais, que se mostraram de bom a excelente, dependendo do período de chuva avaliado. Langeland et al. (2002), Martins et al. (2002) e Cardoso et al. (2003), estudando os efeitos de diquat em plantas de aguapé sem a ocorrência de chuva após sua aplicação, em diferentes doses do herbicida, observaram controles semelhantes aos ora verificados. Esse fato evidencia que, mesmo com a ocorrência de chuva em diferentes períodos após a aplicação de diquat (600 $\mathrm{g}^{\mathrm{i} . \mathrm{a} \mathrm{ha}} \mathrm{h}^{-1}$ ), este herbicida apresentou controles semelhantes aos obtidos em estudos sem a posterior ocorrência de chuva no controle de plantas de E. crassipes.

Considerando os resultados obtidos para as três espécies, observou-se que o herbicida diquat proporcionou excelente controle de $S$. auriculata, $P$. stratiotes e E. crassipes, independentemente do período de tempo para ocorrência de chuva após sua aplicação, até mesmo com ocorrência de chuva imediatamente após sua aplicação.

\section{LITERATURA CITADA}

ANDERSON, M. D.; ARNOLD, W. E. Weed control in sunflowers (Helianthus annuus) with desmediphan and phenmediphan. Weed Sci., v. 32, p. 310-314, 1984.

CAMPOS, C. F. et al. Efeito da chuva sobre a ação do herbicida imazapyr no controle de plantas daninhas aquáticas. Irriga, v. 15, n. 2, p. 151-158, 2010.

CANCIAN, L. F. et al. Crescimento de Pistia stratiotes em diferentes condições de temperatura e fotoperíodo. Acta Bot. Bras., v.23, n.2, p. 552-557, 2009. 
CARDOSO, L. R.; MARTINS, D.; TERRA, M. A Sensibilidade a herbicidas de acessos de aguapé coletados em reservatórios do Estado de São Paulo. Planta Daninha, v. 21, p. 61-67, 2003. (Número Especial)

HENARES, M. N. P. et al. Toxicidade aguda e efeitos histopatológicos do herbicida diquat na brânquia e no fígado da tilápia nilótica (Oreochromis niloticus). Acta Sci. Biol. Sci., v. 30, n. 1, p. 77-82, 2008 a.

HENARES, M. N. P. et al. Toxicidade aguda e efeitos histopatológicos do diquat na brânquia e no fígado do piauçu (Leporinus macrocechalus). Pesticidas, v. 17, p. 107-116, 2007.

HENARES, M. N. P. et al. Toxicidade aguda e histopatologia do herbicida Reward na brânquia e no fígado do pacu (Piaractus mesopotamicus). J. Braz. Soc. Ecotoxicol., v. 3, n. 1 , p. $41-45,2008 b$

INSTITUTO BRASILEIRO DE MEIO AMBIENTE E DOS RECURSOS NATURAIS RENOVÁVEIS - IBAMA. In: WORKSHOP CONTROLE DE PLANTAS AQUÁTICAS, 1988, Brasília. Resumos... Brasília: 1998. p. 1-3.

JAKELAITIS, A. et al. Controle de Digitaria horizontalis pelos herbicidas glyphosate, sulfosate e glifosate potássico submetidos a diferentes intervalos de chuva após a aplicação. Planta Daninha, v. 19, n. 2, p. 279-285, 2001.

KISSMANN, K. G. Plantas infestantes e nocivas. 2.ed. São Bernardo do Campo: BASF, 1997. 852 p.

LANGELAND, K. A. et al. Evaluation of a new formulation of reward landscape and aquatic herbicide for control of duckweed, waterhyacinth, waterlettuce, and hydrilla. J. Aquat. Plant Manag., v. 40, n. 1, p. 51-53, 2002.
LAVORENTI, A. Comportamento dos herbicidas no meio ambiente. In: WORKSHOP SOBRE BIODEGRADAÇÃO, 1996, Campinas. Anais... Jaguariúna: Embrapa-CNPMA, 1996. p. $81-92$

MARCHI, S. R. Efeito de pontas de pulverização e de arranjos populacionais de plantas de Eichhornia crassipes (Mart.) Solms., Salvinia auriculata Aubl. e Pistia stratiotes L. na deposição de calda de pulverização. 2006. 67 f. Tese (Doutorado em Agricultura) - Universidade Estadual Paulista, Botucatu, 2006.

MARTINS, D. et al. Controle químico de Pistia stratiotes, Eichhornia crassipes e Salvinia molesta em caixas d'água. Planta Daninha, v. 20, p. 83-88, 2002. (Número Especial)

MOTTA, M. et al. Avaliação da qualidade da água dos manaciais na ilha de Fernando de Noronha. Ambi-Água, v. 3, n. 3, p. 114-127, 2008.

NEVES, T.; FOLONI, L. L.; PITELLI, R. A. Controle químico do aguapé (Eichhornia crassipes). Planta Daninha, v. 20, p. 89-97, 2002. (Número Especial)

RITTER, A. M. et al. Characterizing aquatic ecological risks from pesticides using a diquat dibromide case study I. Probabilistic exposure estimates. Environ. Toxicol. Chem. v. 19, n. 2 , p. $749-759,2000$

RODRIGUES, B. N.; ALMEIDA, F. S. Guia de herbicidas. 5.ed. Londrina: 2005. 592 p.

SOCIEDADE BRASILEIRA DA CIÊNCIA DAS PLANTAS DANINHAS - SBCPD. Procedimentos para instalação, avaliação e análise de experimentos com herbicidas Londrina: 1995. 42 p. 